\title{
Research Note \\ On the lithium content of the globular cluster M 92
}

\author{
P. Bonifacio * \\ Istituto Nazionale di Astrofisica - Osservatorio Astronomico di Trieste, Via G. B. Tiepolo 11, 34131 Trieste, Italy \\ Received 14 May 2002 / Accepted 9 September 2002

\begin{abstract}
I use literature data and a new temperature calibration to determine the Li abundances in the globular cluster M 92. Based on the same data, Boesgaard et al. have claimed that there is a dispersion in $\mathrm{Li}$ abundances in excess of observational errors. This result has been brought as evidence for Li depletion in metal-poor dwarfs. In the present note I argue that there is no strong evidence for intrinsic dispersion in Li abundances, although a dispersion as large as 0.18 dex is possible. The mean $\mathrm{Li}$ abundance, $A(\mathrm{Li})=2.36$, is in good agreement with recent results for field stars and TO stars in the metal-poor globular cluster NGC 6397. The simplest interpretation is that this constant value represents the primordial Li abundance.
\end{abstract}

Key words. diffusion - stars: abundances - stars: atmospheres - stars: population II - Galaxy: globular clusters: individual: M 92 - cosmology: observations

\section{Introduction}

The present note is motivated by the recent analysis of the metal-poor globular cluster NGC 6397 by Bonifacio et al. (2002). We have confirmed that all the cluster stars share the same Li abundance that is found exactly at the level of the Spite plateau defined for field stars. This supports the notion that $\mathrm{Li}$ in metal-poor stars is of primordial origin and good agreement is found with the other primordial nuclei D and ${ }^{3} \mathrm{He}$. However, in this picture, the observations of Boesgaard et al. (1998) of $\mathrm{Li}$ in the globular cluster M 92 are troublesome. After a careful analysis of the best available data, the above authors conclude that there is a dispersion in $\mathrm{Li}$ abundances by a factor of three, unexplained by observational errors. The most likely explanation suggested for this dispersion is differential Li depletion due to rotational mixing; the stars would deplete more or less $\mathrm{Li}$ depending on their initial angular momentum. While this is a reasonable explanation, one is left to wonder why such effect should be observable in M 92 but not in NGC 6397, which has a very similar metallicity. Moreover, recent models that predict Li depletion either through rotational mixing (Pinsonneault et al. 2002), or diffusion (Salaris \& Weiss 2001), or a combination of both plus composition gradient (Théado \& Vauclair 2001), predict a mild depletion of 0.1-0.2 dex accompanied by a very tiny dispersion. It is doubtful that such a tiny dispersion could be detected in M 92 with the presently available data. The experience with NGC 6397 has shown that use of a photometric

\footnotetext{
* e-mail: bonifaci@ts.astro.it
}

colour such as $b-y$ or $B-V$ to estimate effective temperatures is not well suited to the issue of the scatter in $\mathrm{Li}$ abundances. The $\mathrm{Li}$ abundance is very sensitive to errors in $T_{\text {eff }}$, therefore quite small photometric errors in the colours, translate into large errors on Li abundances. In the case of NGC 6397, Bonifacio et al. (2002) took advantage of the fact that above the TO, where the observed stars lie, there is a tight relation between $V$ magnitude and $b-y$; after correcting for the cluster reddening, this may be calibrated against temperature. The advantage is that an error of 0.05 mag in $V$ translates into an error of only $20-70 \mathrm{~K}$ in $T_{\text {eff }}$. A possible drawback is the vulnerability of the method to binarity and Boesgaard et al. (1998) note that SH 18 may have a variable radial velocity, indicating the presence of a companion. In this note I use the $V-T_{\text {eff }}$ calibration to reanalyse the Li data in M 92.

\section{Analysis}

I take the equivalent widths of Boesgaard et al. (1998). In order to use the $V-T_{\text {eff }}$ calibration of Bonifacio et al. (2002), the cluster fiducial line in the $V,(b-y)_{0}$ plane is required. To assess the scatter in $\mathrm{Li}$ abundances, a transformation of the the fiducial line of the cluster of Stetson \& Harris (1988) from the $(B-V), V$ plane to the $(b-y)_{0}, V$ plane will suffice. For the cluster I adopt the reddening $E(B-V)=0.025$ of Carretta et al. (2000). A transformation from $(b-y)$ to $(B-V)$ has been published by Cousins (1987), however this cannot be readily inverted since it involves also the metallicitydependent index $m_{0}$. To derive a transformation that applies to the TO stars of M 92 I selected the stars used by Bonifacio et al. (2000) to determine their intrinsic colour calibration, which 
Table 1. Equivalent widths and $\mathrm{Li}$ abundances for TO stars in M 92.

\begin{tabular}{lrccrrrrrr}
\hline \hline star & $E W$ & $\sigma_{E W}$ & $T_{\text {eff }}$ & $A(\mathrm{Li})$ & $\sigma_{\mathrm{Li}}$ & $\begin{array}{r}A(\mathrm{Li}) \\
\mathrm{TC}\end{array}$ & $\begin{array}{r}A(\mathrm{Li}) \\
\text { NLTE }\end{array}$ & $\begin{array}{r}A(\mathrm{Li}) \\
\text { B98 (K93) }\end{array}$ & $\begin{array}{r}A(\mathrm{Li}) \\
\text { B98 (C83) }\end{array}$ \\
& $\mathrm{pm}$ & $\mathrm{pm}$ & $\mathrm{K}$ & & & $(5)$ & $(9)(10)$ & \\
\hline SH 18 & 5.93 & 0.69 & 6050 & 2.53 & 0.07 & 2.55 & 2.57 & 2.57 & 2.45 \\
SH 21 & 2.50 & 0.62 & 5921 & 2.00 & 0.13 & 2.04 & 2.06 & 2.06 & 1.96 \\
SH 34 & 5.40 & 1.41 & 5956 & 2.38 & 0.17 & 2.42 & 2.43 & 2.36 & 2.22 \\
SH 46 & 2.87 & 0.74 & 6295 & 2.34 & 0.14 & 2.34 & 2.36 & 2.17 & 2.04 \\
SH 60 & 4.08 & 1.79 & 6171 & 2.51 & 0.23 & 2.52 & 2.54 & 2.42 & 2.30 \\
SH 80 & $<2.20$ & 1.10 & 6557 & $<2.39$ & 0.34 & & & $<2.23$ & $<2.16$ \\
SH 350 & 2.88 & 1.17 & 6102 & 2.20 & 0.25 & 2.22 & 2.23 & 2.13 & 2.01 \\
\hline
\end{tabular}

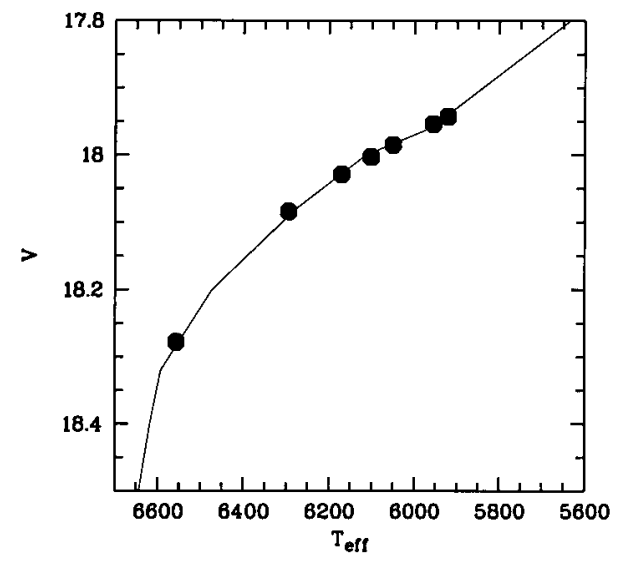

Fig. 1. The adopted $V-T_{\text {eff }}$ calibration: the solid line is the fiducial sequence for M 92, filled symbols correspond to the star in Table 1.

have both Johnson and Strömgren photometry with $-2.5 \leq$ $[\mathrm{Fe} / \mathrm{H}] \leq-1.5$. The data for these stars is summarized in Table 2; the photometric data has been slightly revised with respect to Bonifacio et al. (2000) according to the current holdings of the General Catalog of Photometric data (Mermillod et al. 1996). Both synthetic colours and Cousin's calibration suggest that any transformation between $(B-V)$ and $(b-y)$ should involve a metallicity-dependent term. Since the Johnson system, unlike the Strömgren, does not have any specific metallicity dependent index and the dependence of $U-B$ on metallicity in this range of $B-V$ is as small as about 0.04 mag per 0.5 dex in metallicity, I prefer to use $[\mathrm{Fe} / \mathrm{H}]$ directly as a parameter. With as few as 11 calibrating points, it is not wise to fit anything more complex than a linear relation; however the theoretical colours suggest that this should be adequate. A $\chi^{2}$ fit of a linear relation, taking into account errors in $B-V,[\mathrm{Fe} / \mathrm{H}]$ (a 0.2 dex error has been assumed for all the stars) and $b-y$ provides

$(b-y)=0.74(B-V)-0.05[\mathrm{Fe} / \mathrm{H}]-0.08 ;$

the reduced $\chi^{2}$ is 0.88579 which denotes a good fit and corresponds to a probability of 0.527 , the root mean square is $0.01 \mathrm{mag}$. In the case of $\mathrm{M} 92,[\mathrm{Fe} / \mathrm{H}] \approx-2.0$ so that the transformation may be simplified to

$(b-y) \approx 0.74(B-V)+0.00149$.

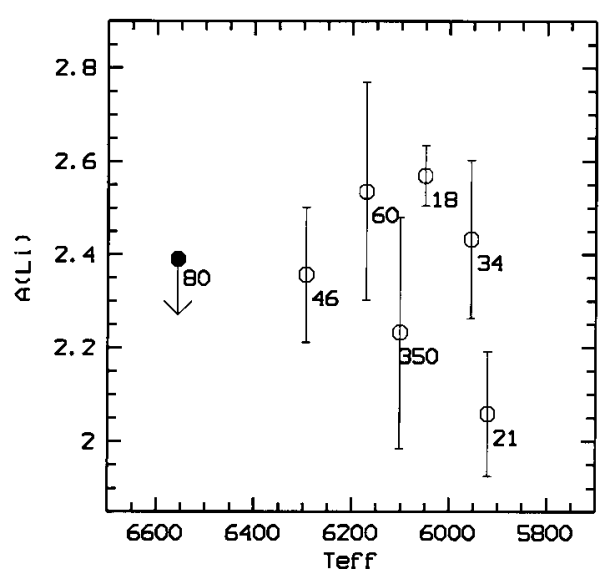

Fig. 2. Li abundances, corrected for standard depletion and NLTE effects, versus effective temperature for TO stars in M 92. The points are labelled with the star's $\mathrm{SH}$ number.

This calibration is formally valid only for the colour and metallicity range spanned by the calibrators, thus $0.357 \leq B-V \leq$ 0.487. Three of the stars observed by Boesgaard et al. (1998) are slightly cooler than this range, however the largest extrapolation required is for star \#34 and it is of only $0.031 \mathrm{mag}$, and thus is not a serious problem for the issue addressed in this note.

I use the above transformation to transpose the fiducial TO of Stetson \& Harris (1988) to the $(b-y)_{0}, V$ plane. From this I obtain the $V-T_{\text {eff }}$ calibration using the same $b-y-T_{\text {eff }}$ used in Bonifacio et al. (2002). With these effective temperatures I derive Li abundances, as described in Bonifacio et al. (2002): I computed a model atmosphere for each temperature and $\log g=3.75$, as adopted by King et al. (1998). The internal errors in effective temperature arising from the the photometric error in $V$ are of the order of 10 to $50 \mathrm{~K}$ and can be neglected in estimating the error on $A(\mathrm{Li})^{1}$, which is totally dominated by the errors on the equivalent widths. Of course the external error is at least of the order of $100 \mathrm{~K}$, and possibly larger, since I did not use Strömgren photometry; however for the purpose of the present discussion I shall not consider the external error on effective temperatures. Effective temperatures, Li abundances

\footnotetext{
${ }^{1}$ I use the usual notation $A(\mathrm{Li})=12+\log [N(\mathrm{Li}) / N(\mathrm{H})]$.
} 
Table 2. Data for the field stars used to derive the transformation.

\begin{tabular}{lccccc}
\hline \hline star & $B-V$ & $\sigma_{B-V}$ & $b-y$ & $\sigma_{b-y}$ & {$[\mathrm{Fe} / \mathrm{H}]$} \\
\hline G165-39 & 0.404 & 0.013 & 0.309 & 0.001 & -2.05 \\
G88-32 & 0.357 & 0.005 & 0.309 & 0.006 & -2.36 \\
G8-16 & 0.424 & 0.020 & 0.322 & 0.006 & -1.59 \\
HD 16031 & 0.441 & 0.003 & 0.323 & 0.005 & -1.71 \\
G13-35 & 0.432 & 0.007 & 0.331 & 0.003 & -1.63 \\
G59-24 & 0.423 & 0.008 & 0.332 & 0.006 & -2.42 \\
G11-44 & 0.434 & 0.011 & 0.334 & 0.001 & -2.07 \\
G114-26 & 0.481 & 0.007 & 0.349 & 0.006 & -1.78 \\
G37-26 & 0.459 & 0.009 & 0.351 & 0.003 & -1.93 \\
G60-48 & 0.487 & 0.004 & 0.365 & 0.005 & -1.63 \\
HD 34328 & 0.486 & 0.006 & 0.365 & 0.007 & -1.61 \\
\hline
\end{tabular}

with their errors, as the equivalent widths of Boesgaard et al. (1998) as well as their Li abundances both on the King (1993) and on the Carney (1983) scale, are given in Table 1.

There are 6 measures and one upper limit. Not unexpectedly the upper limit is for the hottest and faintest star of the sample, therefore the one with the smallest equivalent width of the Li doublet and among the smallest $S / N$ ratios. The mean $A(\mathrm{Li})$ for the six measurements is 2.33 with a standard deviation of 0.20. In order to assess if this scatter is compatible with the observational error I use a Monte Carlo simulation, as done by Bonifacio et al. (2002). I generate 10000 samples of 7 "observations", each scattered around a mean 2.3 with a Gaussian distribution of $\sigma$ equal to the estimated error in $A(\mathrm{Li})$ and compute the standard deviation $s$ of these 7 "observations". For the upper limit I consider an error of $0.34 \mathrm{dex}$, which is the error corresponding to an error in equivalent width of $1.1 \mathrm{pm}$ for a star of $T_{\text {eff }}=6557 \mathrm{~K}$. The mean $s$ of the 10000 samples is 0.20 with a standard deviation of 0.07 , therefore the simulations suggests that the observed scatter is entirely due to the errors in the observations. I can also estimate the probability of having a non-detection of $\mathrm{Li}$ in at least one star of the sample of 7. I define the minimum $A(\mathrm{Li})$ detectable as 1.72 , based on the data for $\mathrm{SH} 80$, and find that out of 10000 samples, 400 satisfy this condition, therefore the probability of having a non-detection is about $4 \%$. This probability is small but nonnegligible. Of course since the errors are rather large (due to the faintness of the TO of M 92) there could be an intrinsic scatter in $\mathrm{Li}$ abundances of the order of $0.18 \mathrm{dex}$, which would go totally unnoticed. One could suspect that the scatter found in the Monte Carlo simulations is dominated by the inclusion of the rather large error associated with star $\mathrm{SH} 80$, however this is not the case. I performed a Monte Carlo simulation with only 6 "observations" and the mean $s$ is 0.17 with a standard deviation of 0.06 , therefore although the scatter has undoubtedly decreased, at $1 \sigma$ this is consistent with the observed scatter. One could further argue that the lack of detection of any extra scatter is the result of considering also stars which have a very poor Li abundance determination. If we, somewhat arbitrarily, select only the stars for which the error on $A(\mathrm{Li})$ is less than 0.2 dex we are left with only 4 stars. The mean $A(\mathrm{Li})$ is 2.31 ,
Table 3. Li curve of growth for $T_{\text {eff }}=5951, \log g=3.75,[\mathrm{Fe} / \mathrm{H}]=$ -2.0 .

\begin{tabular}{rrr}
\hline \hline$E W(\mathrm{pm})$ & $\log (E W)$ & $A(\mathrm{Li})$ \\
\hline 2.09 & 0.320146 & 1.94 \\
2.13 & 0.328380 & 1.95 \\
2.64 & 0.421604 & 2.05 \\
2.87 & 0.457882 & 2.09 \\
3.61 & 0.557507 & 2.21 \\
5.18 & 0.714330 & 2.39 \\
8.00 & 0.903090 & 2.64 \\
\hline
\end{tabular}

not significantly changed, and the dispersion is slightly larger: 0.22 dex. A Monte Carlo simulation with 4 "stars" provides a mean standard deviation, over 10000 samples, of the four "observations" $<s>=0.12$ with a standard deviation of 0.05 dex. Now an excess dispersion is detected, but at $2 \sigma$ only. At three $\sigma$ there is no evidence of extra dispersion. One could claim that the existence of "intrinsic dispersion" is implied by the observation of SH 18 and SH 21, whose Li abundance differs by 0.53 dex. In fact the two measures are compatible at $2.7 \sigma$. We may obtain an estimate of how likely this is by looking at the Monte Carlo simulation: out of 10000 samples in four realizations the two "stars" differ by more than this amount (in fact on one occasion they differ by $0.65 \mathrm{dex}$ ). This is a small probability; however whether one considers this negligible or not must depend also on other information.

If we perform the corrections due to standard depletion and NLTE, as described in Bonifacio et al. (2002), the scatter in the data is diminished by very little $(0.19$ dex rather than $0.20)$. However the mean $A(\mathrm{Li})$ is now 2.36. This value is in good agreement with the Li abundance of field stars (Bonifacio 2001) and of TO stars in NGC 6397 (Bonifacio et al. 2002). The purpose of adopting the $V, T_{\text {eff }}$ calibration was to make a direct comparison between M 92 and NGC 6397, however the dispersion is not decreased by the adoption of this temperature scale, in fact the Li abundances of Boesgaard et al. (1998) have a scatter of 0.20 dex on the King (1993) scale and 0.19 dex on the Carney (1983) scale, essentially the same as found using the $V, T_{\text {eff }}$ scale. Performing a Monte Carlo simulation using 6 "observations" with the errors of Boesgaard et al. (1998) I obtain a mean $s$ of 0.12 dex with a standard deviation of 0.05 dex for the King (1993) scale and 0.13 dex with a standard deviation of 0.05 dex for the Carney (1983) scale. In this case extra dispersion is detected at $1 \sigma$ in both cases, but in neither case at $2 \sigma$. The lower scatter is due to the smaller errors adopted by Boesgaard et al. (1998) with respect to mine for several stars. This is odd, since Boesgaard et al. (1998) add quadratically the errors due to equivalent widths and effective temperatures, whereas I consider only the error due to the equivalent widths. Therefore, my errors should be, if anything, smaller. The only possible explanation is that there are some typographical errors in Table 2 of Boesgaard et al. (1998). To check this I computed the curve of growth using a model with $T_{\text {eff }}=5951 \mathrm{~K}$, which is adopted by Boesgaard et al. (1998) for SH 46 on the King (1993) scale. This is given in Table 3. From this table it can be 
seen that an error of $0.74 \mathrm{pm}$ in equivalent width, around an equivalent width of $2.87 \mathrm{pm}$ induces an error of $0.14 \mathrm{dex}$ on the Li abundance. The error given in Table 2 of Boesgaard et al. (1998) is 0.040 for the King (1993) scale and I think it is likely to be a typographical error.

\section{Conclusions}

From this reanalysis of existing data of M 92 I conclude that there is no strong support for an intrinsic dispersion in $\mathrm{Li}$ abundances; however, a dispersion of the order of 0.18 dex would go unnoticed with the present errors. The mean $\mathrm{Li}$ abundance is in good agreement with that found for field stars and for TO stars of NGC 6397, which has a metallicity comparable to that of M 92. I believe that the simplest hypothesis is that $\mathrm{Li}$ is constant in M 92 and at the level of the Spite plateau. However, the main purpose of this note is to encourage observers to obtain spectra with higher $S / N$ ratio in order to check if this is really the case. It is clear that statistical analysis and Monte Carlo simulations cannot make up for inadequate observations. The presently-available observations of M 92 cannot prove nor disprove the existence of an "intrinsic" dispersion. In particular, if SH 80 is really Li-depleted, it would be the first Li-depleted dwarf so-far detected in a metal-poor globular cluster and could testify in favour of Li-depletion mechanisms in metal-poor dwarfs. It is also obvious that an instrument like HIRES cannot tackle the problem without a prohibitive investment of telescope time. The new generation of medium resolution spectrographs operating on $8 \mathrm{~m}$ telescopes, like ESI at Keck (Epps \& Miller 1998), or proposed, like AVES for VLT (Pallavicini et al. 2000), have the capability to solve the problem. For example, ESI, with an entrance slit of 0.3 which projects to 1.95 pixels, provides a resolving power of $0.032 \mathrm{~nm}$ per resolution element, which does not fully resolve the Li doublet, but is enough to measure an accurate equivalent width. There is no exposure time calculator for ESI, but a $V=10.55$ star provides $56650 \mathrm{e}^{-} \mathrm{nm}^{-1} \mathrm{~s}^{-1}$; scaling this number for a $V=$ 18.278 star ( $\mathrm{SH} \mathrm{80)}$ one obtains $46 \mathrm{e}^{-} \mathrm{nm}^{-1} \mathrm{~s}^{-1}$, therefore in one hour of exposure the $S / N \sim 73$ per resolution element. Assuming one can reach a $S / N \sim 140$ adding 4 one hour exposures the Cayrel formula (Cayrel 1988) provides an error on the equivalent width of $0.24 \mathrm{pm}$, i.e. comparable to what was obtained by Bonifacio et al. (2002) for the much brighter stars in NGC 6397. AVES has been proposed for the VLT and could not obviously observe M 92; however the design of AVES can easily be modified for a different telescope and its performances without adaptive optics would be similar to those of ESI and better with adaptive optics. Therefore it is from these medium resolution spectrographs that we must expect accurate abundances for TO stars in Galactic Globular Clusters in the near future.

Acknowledgements. This work was done while I was at the Observatoire de Paris-Meudon as a visitor. R. Cayrel, M. Spite and F. Spite are warmly thanked for many helpful discussions. I am grateful to the referee, P. E. Nissen, for pointing out the need for a colourcolour transformation valid for metal-poor stars. This research was done with support from the italian MURST/MIUR COFIN2000 grant "Spettroscopia e studio dei processi fisici del Sole e delle stelle di tipo solare" (P. I. G. Peres).

\section{References}

Boesgaard, A. M., Deliyannis, C. P., Stephens, A., \& King, J. R. 1998, ApJ, 493, 206

Bonifacio, P., Caffau, E., \& Molaro, P. 2000, A\&AS, 145, 473

Bonifacio, P. 2001, in The link between stars and cosmology, ed. M. Chavez, \& D. Mayya (Dordrecht: Kluwer), in press

Bonifacio, P., et al. 2002, A\&A, 390, 91

Carney, B. W. 1983, AJ, 88, 610

Carretta, E., Gratton, R. G., Clementini, G., \& Fusi Pecci, F. 2000, ApJ, 533, 215

Cayrel, R. 1988, in The Impact of Very High $S / N$ Spectroscopy on Stellar Physics, ed. G. Cayrel de Strobel, \& M. Spite (Dordrecht: Kluwer), 345

Cousins, A. W. J. 1987, The Observatory, 107, 80

Epps, H. W., \& Miller, J. S. 1998, Proc. SPIE, 3355, 48

King, J. R. 1993, AJ, 106, 1206

King, J. R., Stephens, A., Boesgaard, A. M., \& Deliyannis, C. 1998, AJ, 115, 666

Mermillod, J. C., Hauck, B., \& Mermillod, M. 1996, General Catalog of Photometric Data

http://obswww. unige.ch/gcpd/gcdp.html

Pallavicini, R., et al. 2000, Proc. SPIE, 4008, 167

Pinsonneault, M. H., Steigman, G., Walker, T. P., \& Narayanan, V. K. 2002, ApJ, 574, 398

Salaris, M., \& Weiss, A. 2001, A\&A, 376, 955

Stetson, P. B., \& Harris, W. E. 1988, AJ, 96, 909

Théado, S., \& Vauclair, S. 2001, A\&A, 375, 70 